\title{
Normal erythropoiesis and development of multiple myeloma
}

\author{
A. Bouchnita ${ }^{1,2, a}$, N. Eymard ${ }^{2,}$, M. Koury ${ }^{3}$, T. Moyo ${ }^{3}$, and V. Volpert ${ }^{2}$, \\ ${ }^{1}$ Laboratoire de Biométrie et Biologie Evolutive (LBBE), University Lyon 1 \\ 69622 Villeurbanne, France \\ ${ }^{2}$ Institut Camille Jordan (ICJ), University Lyon 1 \\ 69622 Villeurbanne, France \\ ${ }^{3}$ Vanderbilt University Medical Center, Nashville, TN, USA
}

\begin{abstract}
We develop a hybrid multi-scale model of normal and pathological erythropoiesis, the process of red blood cell production in the bone marrow. Cells are described by a soft sphere model. They can move, divide and die by apoptosis. Their fate is determined by intracellular proteins, by extracellular substances and surrounding cells. We apply this model to describe normal function of erythropoiesis and the development of multiple myeloma in the bone marrow. It is a malignant disease characterized by proliferation of mutated plasma cells which form multiple tumors in the bone marrow. They disrupt erythroblastic islands by direct mechanical contact and due to apoptotic cytokines produced by myeloma cells. The development of the disease leads to decrease of red blood cell production and to anemia.
\end{abstract}

\section{Introduction}

Erythropoiesis is the process of red blood cells production. In adult humans it mainly occurs in the bone marrow in erythroblastic islands (EIs). These are multi-cellular structures that represent the primary units of erythropoiesis. The EI is composed by both early and late erythroblasts surrounding a central macrophage. The latter plays a central role in promoting the proliferation of CFUE/ProEbs cells [1, 2]. After describing normal erythropoiesis, we will model the development of multiple myeloma (MM). It is characterized by the infiltration of malignant myeloma cells in the bone marrow. These cells proliferate and destroy erythroblastic islands by pushing the erythroid cells away from central macrophages. They also secrete apoptosis-inducing cytokines (FasL and TRAIL) [1]. These two effects lead to the insufficient production of red blood cells and to anemia.

\section{The model}

We present here a hybrid model developed in order to study normal and pathological erythropoiesis [3]-[6]. It includes cell based modelling with intracellular and extracellular regulation of cell fate. Each CFU-E/ProEb cell has two different possible fates: it will either die by apoptosis or it will survive and divide. If it divides, then it will either give birth to two CFU-E/ProEbs (auto-renewal) or

\footnotetext{
ae-mail: anass.bouchnita@univ-lyon1.fr
} 
two daughter cells of a later differentiation stage (differentiation). Each one of these possible fates is regulated by the concentrations of intracellular proteins. The latter are affected by surrounding cells and some proteins in the extracellular matrix.

\subsection{Intracellular regulation}

The fate of early erythroblasts is regulated by intracellular proteins. We use a system of four ordinary differential equations to describe the evolution of the concentration of each protein inside the cell:

$$
\frac{d z}{d t}=k_{\text {new }}, \quad \frac{d u}{d t}=\gamma_{1}+b_{0}, \quad \frac{d v}{d t}=\gamma_{2}-b_{2} z v \quad \frac{d w}{d t}=\gamma_{3}-b_{3} z w .
$$

Here $z$ is the concentration of glucocorticosteroid receptors (GR), a protein that stimulates the autorenewal of the cell by inhibiting its differentiation or apoptosis; $u$ is the concetration of activated BMPR, a protein that is the receptot for BMP4 and stimulates self-renewal, BMP4 is secreted by the central macrophages. Another important protein is GATA-1, whose concentration is denoted by $v$. GATA-1 is promoted by exterior FasL or TRAIL that are secreted by either the late erythroblasts or myeloma cells. GATA1 is an erythroid transcription factor that induces differentiation if $u<v$ at the time of cell division of the CFU-E/ProEB. The CFU-E/ProEB auto-renews otherwise. Lastly, caspase $w$ is an intracellular protein whose concentrations induce the erythroblast apoptosis if they exceed a threshold $w_{c r}$.

\subsection{Extracellular regulation}

The intracellular protein network is regulated by extracellular proteins concentrations. In our model, we consider only main two proteins which are FasL $F$, an apoptosis-inducing cytokine, and a growth factor $\mathrm{KL} / \mathrm{SCF} G$. We use reaction-diffusion equations to describe the concentrations of these proteins in the extracellular matrix:

$$
\begin{aligned}
& \frac{\partial F}{\partial t}=D_{F} \Delta F+W_{1}-\sigma_{1} F, \\
& \frac{\partial G}{\partial t}=D_{G} \Delta G+W_{2}-\sigma_{2} G,
\end{aligned}
$$

where $D_{F}$ and $D_{G}$ are the diffusion coefficients of $F_{L}$ and $G$ respectively, $W_{1}$ and $W_{2}$ are the production rates of these two proteins. The last terms in the right-hand sides of these equations describe their degradation.

\subsection{Cell motion and division}

Erythroid cells do not move by themselves but they can change their position when they are pushed by other cells. We model them as elastic spheres and describe their motion with Newton's second law for their centers. Before division, the erythroid cell increases its size and then divides into two daughter cells with smaller radii. More detail on the cell based modelling of erythropoiesis can be found in $[4,5]$. 


\section{Normal erythropoiesis}

A typical structure of an erythroblastic island is shown in Figure 1 (left). The central macrophage (big cell in the center) is surrounded by erythroid progenitors (yellow cells). Progenitors make a choice between self-renewal, differentiation and apoptosis. This choice is determined by the intracellular regulation, and it depends on the extracellular substances, growth factors produced by the macrophage and Fas-ligand produced by the mature erythroblasts and the reticulocytes formed from them. Progenitors located close to the macrophage self-renew due to the growth factor. Progenitors close to reticulocytes die by apoptosis due to Fas-ligand. Progenitors located in between differentiate, eventually becoming reticulocytes.
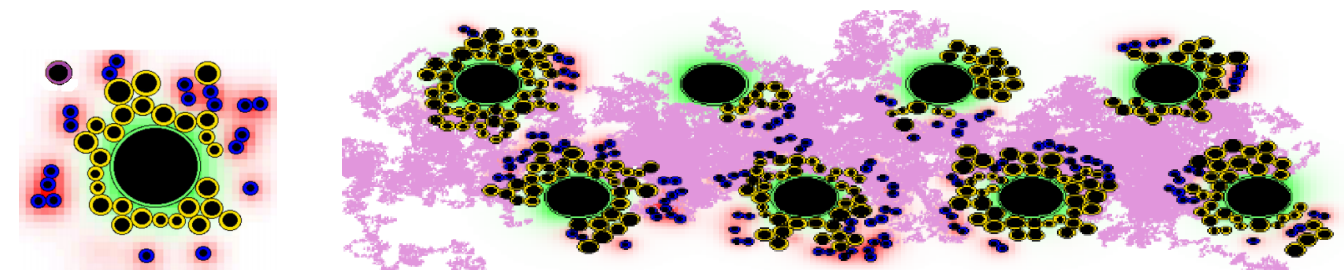

Figure 1. Left: the structure of erythroblastic island. The green large cell in the middle is the central macrophage. Yellow cells are erythroid progenitors (CFU-E/erythroblasts), blue cells are reticulocytes, the violet cell is BFUE. The red substance secreted by reticulocytes is Fas-Ligand. Growth factor produced by the macrophage is shown in green. Black circles inside cells show their incompressible part. Right: burst forming unit (BFU-E) cells move randomly in the bone marrow. Magenta curve behind the cell shows its trajectory. When the cell approaches a macrophage (big cell), it divides and gives birth to two CFU-Es (erythroid progenitor). Progenitors divide creating an erythroblastic island around the macrophage.

After some time, reticulocytes leave the bone marrow into blood flow where they become mature erythrocytes. Since EIs consist of a small number of cells, random perturbations in cell cycle duration, apoptosis of erythroid progenitors and departure of erythrocytes can lead to extinction of the islands. This means that the macrophage remains without erythroid progenitors around it, and new reticulocytes are not produced. Hence there should exist a mechanism of reinitiation of erythroblastic islands. Moreover, in the case of stress erythropoiesis (anemia, hypoxia) the number of erythroblastic islands can rapidly increase. This reinitiation mechanism is provided by BFU-E cells. They move randomly in the bone marrow. When they meet a macrophage, they divide producing CFU-Es and erythroid progenitors thereby restarting the erythroblastic island.

In order to study this effect, we consider several macrophages without erythroid progenitors and introduce BFU-E cells (Figure 1, left). The BFU-Es move in the bone marrow in a random way. Their trajectories are shown by magenta curves. When they approach the macrophages, they are influenced by the growth factor and begin to proliferate producing two CFU-Es. On the other hand, the erythroblasts lose their adherence to the central macrophage, allowing them to be pushed out by other cells. As we discussed above, CFU-Es self-renew or differentiate, restarting the extinct island or forming a new one. 


\section{Multiple myeloma development}

Multiple myeloma develops in the bone marrow due to abnormal proliferation of mutated plasma cells. They can leave the bone marrow and return back to it with the blood flow. Because of this mobility, they form multiple tumors.
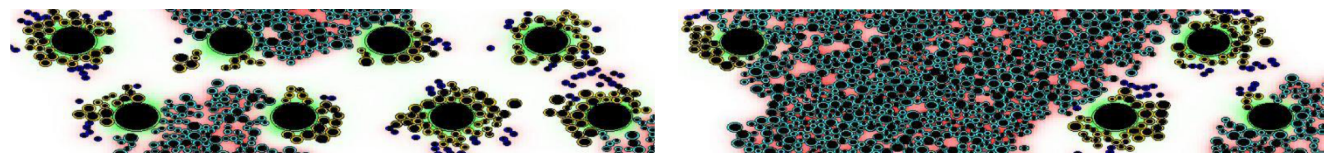

Figure 2. Two stages of the development of multiple myeloma. Myeloma cells form multiple tumors and destroy erythroblastic islands mechanically and biochemically. In the beginning of myeloma development, erythroblastic islands are weakly influenced and continue their functioning (left). After some time, tumor fills an important part of the marrow and all islands are destroyed (right).

In order to model the disease progression, we add myeloma cells to the model of normal erythropoiesis described above [7]. We consider a section of the bone marrow and put there several myeloma cells. They proliferate with a given rate producing similar cells after each division. Myeloma cells influence erythroid cells by two mechanisms. Tumor growth pushes erythroid cells and destroys erythroblastic islands by pure mechanical action. On the other hand, myeloma cells produce Fas-ligand and TRAIL which stimulates apoptosis of erythroid progenitors.

Two stages of myeloma development are shown in Figure 2. In the beginning of its development, erythroblastic islands are weakly influenced and continue their normal functioning. They become more and more influenced by myeloma progression, and finally they are completely destroyed.

Multiple myeloma is treated by chemotherapy that is frequently followed by bone marrow transplantation. Modelling of myeloma treatment and comparison with the clinical data will be presented in the subsequent works.

\section{References}

[1] Beguin, Y. (1995). Erythropoiesis and erythropoietin in multiple myeloma. Leukemia $\mathcal{F}$ lymphoma, 18(5-6), 413-421.

[2] Koury, M. J. (2005) Erythropoietin: the story of hypoxia and a finely regulated hematopoietic hormone. Experimental hematology, 33(11), 1263-1270.

[3] N. Bessonov, N. Eymard, P. Kurbatova, V. Volpert. Mathematical modelling of erythropoiesis in vivo with multiple erythroblastic islands. Applied Mathematics Letters, 25 (2012) 1217-1221.

[4] Eymard, N., Bessonov, N., Gandrillon, O., Koury, M. J., \& Volpert, V. (2014). The role of spatial organization of cells in erythropoiesis. Journal of mathematical biology, 1-27.

[5] Fischer, S., Kurbatova, P., Bessonov, N., Gandrillon, O., Volpert, V., \& Crauste, F. (2012). Modeling erythroblastic islands: using a hybrid model to assess the function of central macrophage. Journal of theoretical biology, 298, 92-106.

[6] P. Kurbatova, N. Eymard, V. Volpert. Hybrid Model of Erythropoiesis. Acta Biotheoretica, 61 (2013), 305-315.

[7] A. Bouchnita, N. Eymard, M. Koury, V. Volpert. Initiation of erythropoiesis by BFU-E cells. ITM Web of Conferences 4, 01002 (2015). 